\title{
12
}

\section{Conclusion: back to the future}

\section{Alex Gekker, Sam Hind, Sybille Lammes, Chris Perkins and Clancy Wilmott}

The chapters in this book have emerged and changed in an ongoing process. You are reading something that is apparently fixed, with an endpoint in terms of its production. But tracing the emerging ideas back to an origin is a much more challenging task; each chapter - and indeed this conclusion - emerged gradually and in a nonlinear fashion. Events can be planned or random, they may be fast or slow and digital tools may allow us to accelerate or impede this process. New digital technologies also afforded us the chance to enact specific rhythms in the writing process. We worked quickly on this book as editors, by organising a series of book-sprints in remote areas in the United Kingdom and the Netherlands, as well as across digital and virtual time-spaces. During this process, we collaborated on editing chapters simultaneously and jointly - composing the conclusion and introduction in shared Google Docs, watching and over-writing each other's work. How we, during a shared yet sometimes haphazard time frame of days, hours or moments, worked on this book serves as a useful allusion to how temporality has taken on new meanings since the advent of digital mapping.

Much like the digital mapping practices we have discussed in this book, time proved to be tricky, asynchronous, serendipitous, sticky and ephemeral, thanks to - or despite - digital technologies. Someone was sent out of the room where she Skyped, someone else had to walk a dog, was hungry and had to grab lunch, forgot about time differences, spilled a drink over a computer, or failed to find the right document. Every time we faced an interruption, we had to try to realign our times so as to recover glimpses of immediacy. The goal was for the palimpsest of an emerging document to become a conceptual landscape, resonating with the sometimes false promises of immediacy that digital mapping so often seems to deliver. 
Based in different countries and universities, we came together in shared spaces to deliver an apparently fixed outcome; akin to how crowd-sourced mapping emerges from differently situated participants. The process of making this book is the result of an effort to synchronise time over different places. It shows how the digital can invite us to be in a different place at the same time, tracking each other's emerging paths from a distance. Yet it also shows how time and technologies can subvert our expectations, lead to unexpected results and otherwise fail. For example, Skype connections on mobile phones picked up background noises which obscured the actual voice of the speaker, and electronically edited documents escaped attention due to the temporal simultaneity of this medium. So the words may be about time but they also offer a glimpse into aspects of time as they emerge from an ongoing process of writing. This process of writing stands in many ways as analogous to mapping, and towards the end of a process, concluding chapters traditionally reflect on what has been delivered and on what the future might entail. Whereas a digital map is never complete (Kitchin and Dodge, 2007), this book is ending. So, looking back and forward - much like a Trip Advisor restaurant review reflecting on a meal, but also inviting others to experience it - we argue that it is about time!

Chapters in this book have explored conceptual frameworks for understanding the rhythms, ephemerality, transformativity, futurity and immediacy of digital mapping. They therefore demonstrate why these temporal concepts are needed to start to make sense of new cartographic forms emerging after the advent of the digital. Be it in art, daily life or planning, data production and consumption continue to accelerate apace, and new affordances emerge for engaging with maps accordingly. Mobility further complicates this unfolding of contemporary cartographies and brings with it new conceptions of temporality, as the map itself and the user are not only on the move, but also reciprocally transform each other through time, processes that are often difficult to grasp.

This book has offered some tools to address these changes. Of course, when temporalities become privileged, this has a reciprocal effect on how we view space. Previously hidden temporal conceptualisations may be needed to understand digital mapping practices, but they do not exist independent of spatiality. The concepts explored in this book communicate ideas, even when they are being used as an approach, and in turn impact on mapping practices. In this conclusion, we take stock and look back at what our temporal foci has done for time-space and its imagining as a process, highlighting questions that have emerged during this process.

This processual quality of temporality is important, but many other ways of approaching time might be deployed. We started our consideration of temporality in the introduction by deploying Barbara Adam's (2008) sevenfold 
classification of time as a means of discussing different philosophical approaches to them. Adam highlights seven features of time: time frame; temporality; timing; tempo; duration; sequence and modalities (for an explanation of their significance see chapter 1 this volume). By examining each section of this collected volume in relation to its emerging internal consistency with Adam's typology, we highlight how different authors foreground different aspects of temporality. In doing so, we suggest aspects that have perhaps been underanalysed and where future research might profitably focus.

\section{Ephemerality/mobility}

Against the grand scales of history, ephemerality and mobility offer useful entry points into a nuanced discussion about everyday temporality, precisely because of the complex questions they pose for mapping. On the one hand, fleeting encounters and irretrievable processes engage mapping as an iterative and temporary practice, that works in both performative and representational ways, and which builds and dissolves according to heterogeneous modalities. On the other hand, mapping 'products' frequently retain a longevity across multiple and ephemeral platforms - such as OpenStreetMap, Google Street View or McLean's smell maps. The ways in which each platform presents a version of temporality is encased in their own unique time frames. Questions arise in this disjunction between the ephemeral (or that which doesn't last), presented by the analysis of encounter by Gerlach, McLean and Abend, and the lingering traces, data sweat (Gregg, 2015) - or data fumes (Thatcher, 2014) - that endure beyond the moment. A key issue is how do we resolve the question of scale when considering temporality? For space and place, we already have some answers - Massey (1991: 24) suggests it is imperative to reconsider places, not as a bounded and defined, but as spaces with histories. This is particularly salient at a time 'when things are speeding up' due to time-space compression and when the global and the local become ever more intimately intertwined. It is a time when 'history itself is imagined as the product of layer-upon-layer of different sets of linkages, both local and to the wider world' (Massey, 1991: 29). But if we were to invert that assertion, as we have tried to do throughout this volume, to focus not on spatio-temporality, but on tempo-spatiality, then a different set of questions emerge.

First, a temporal focus draws attention to multifaceted sets of scale, which appear to braid into different temporal durations at social and personal levels, in an age when time appears to accelerate exponentially. Walter Benjamin once described this as Erfahrung and Erlebnis (the temporal distinctiveness of near 
and far experience and memory) (see Elsaesser, 2009), and the digital mapping cases discussed in this section also evoke Bergsonian notions of durée and Adam's (2008) notion of duration. Gerlach in particular highlights the implications of an approach focusing on these durative, as against chronological or sequential, issues. We can also see this process re-enacted in many examples of memory work, deployed close to hand, but also using digital connections to call into play remote experiences. Tracks and points are employed by the Intel Corporation as a system for data retention in computing, delivering a shared metric for everyone, generalised through GPS models and readily available beneath smartphone interfaces. But this shared cartographic reason (Olsson, 2007) is deployed by individuals whose experiences and memories of experiences are locally enacted and of the moment. As such, a mobile and personal experience of temporality reveals tensions in digital mapping (Wilmott, 2016). Time is global, but that registers and regulates against temporality, which is lived and experienced. These tensions are further explored in this section. Gerlach waits for his GPS device to arrive, and when it does, the device records and reinforces personal narratives at thirty-second intervals. Similarly, McLean's plural mapping processes makes links between people, smells and space, and the end product, a map, which at once challenges what has traditionally been perceived as being cartographic, while at the same time capturing a series of ephemeral moments, a flow, by way of traces on paper. Our third case from Abend recounts the glitches he encounters which fragment and disrupt the uniformity of time ambitiously presented by Google Street View. Once again, the near and the far merge in a technological process. There is a rich potential for research that focuses upon these scalar tensions, and its inherently social focus lies beyond Adam's (2008) classification.

A second set of questions arise because the ephemeral cannot be disentangled from the epochal as easily as we imagine. Digital mapping builds on cartographic traditions that are thoroughly epochal traditions which are structured and scaffolded to scale from the very local and immediate, to the very global and distant. The glimpse of a suburban street-in-action, or the everyday vernacular mappings of a local OpenStreetMap group, or the recording of transient sensation of urban smells, are juxtaposed to massive collections of data accrued through millions of these moments. This scaling delivers the power of maps (Wood, 1992). But it is no longer through the representational fixity of pen on paper, or the temporal selection of the image, and no longer by the aesthetic or artistic practice of cartographers and groups of surveyors employed by nation-states to fix and frame their territories. Instead, the presence of the Google car makes these links possible, and formerly clear distinctions between users and producers blur in the world of crowd-sourcing and the 'gig' economy. A very different digital assemblage comes together to make these links, which makes for changed 
affordances and changing ways in which the temporal is captured. There are further discussions to be had here about these interrelations between heterogeneous temporalities, the relationship between mapping processes and mapping products, and the arbitrary designation of timescales into frames - seconds, minutes, hours, days, weeks, or months - through which we understand time. Once again, the neat classification from Adam breaks down and once again, research is needed to explore the relations that emerge.

A third and crucial question that emerges from this section relates to the temporal layering that is implicit in most digital mapping (see Verhoeff, 2012). Massey (1991) asks how space enrols time, but how can we rethink a temporality which pulls in layers of spaces and places? She describes a historical space, which is the product of interlinked layers of historical moments and events, but layered digital mapping enrolling space into time complicates outcomes. What does it mean to reject the clarity of borders between history and experience, between the global stories that build empires and nations into perpetuity (even if only in the archive), and those that build memories and everyday localities? The precise role that digital mapping might play in this process remains to be charted in detail, beyond the cases suggested in our chapters.

So a focus on ephemerality and mobilities suggests research agendas that are rather more performative, transient and hybrid than might be imagined in the at times static implications of Barbara Adam's (2008) typology.

\section{Stitching memories}

In this section of the book, we drew attention to how the digital has foregrounded an accelerated process of combining and recombining cartographical data, and how tempo has encouraged us to grapple for new ways to remember and capture places and trajectories when it all seems to slip to our fingers so easily. Our authors ask what practices might exist to counter the ephemeralities and mobilities that digital mapping has brought with it, which were discussed in the first section of the book. As we are dealing with assemblages of digital technologies, their constant shifting employment can lead to an impression of time 'flying', and of an inevitable mutability. But the very same technologies can also be aligned and mastered to re-appropriate experiences and to stitch memories together in novel ways. This section was about reflection and looking back, to track what has happened on the map and create 'bricolages' with their own tempos and histories.

Authors in this part of the book discussed daily and creative practices that actually address some of the questions that the first section of the book left us 
with, and especially the role that digital mapping might play in building memories by creating new temporal tapestries. As this section is concerned with the capturing and recombining of different moments and memories, the emphasis shifts from Adam's (2008) categories of duration to tempo; the speed, but also the stillness, that is called into play when slivers of time are recombined, and how such capturing processes are visualised as staccato cartographical fabrics. This stitching inevitably only delivers an edited version of the past: events and experiences can be edited and recombined, forming new patterns of memories, but also frequently hide the editorial processes that were employed to make the stitch-work!

The first two chapters are mostly concerned with artistic interventions that use digital tools to map past time, and find new ways of producing stories through them, much in the same vein as the smell maps of Kate McLean seek to make narrative accounts of smellscapes. However, unlike McLean's work, they empathise the sutures instead of temporal flow. Rachel Wells' chapter about the temporal maps created by visual artist Wolfgang Weileder discusses the reordering of temporalities. In his atlas-project, he stacks slices of photographic renderings of the same place on top of each other to create a visual rendering of different moments stitched together through techniques of digital editing. Likewise, MacDonald shows how art projects can capture mapping processes by recounting mapping moments in the past and bringing them together in new narratives, thus showing how remnants of past mapping processes can form new productive relations for remembering place. Such attempts are not exclusive to the realm of artistic practice though, but can also be found in daily life, as Hanchard shows in his chapter. Advocating a practice theory perspective on quotidian mapping, he shows an intricate patchwork of stories through which people remember and reflect on quotidian mapping practices. So, by stressing the temporal dimension of cartographical stitching of memories and stories, we have showed here some counter-strategies to the ephemeral quality of digital mapping.

The first question that arises from these chapters concerns how successful these strategies might be for facilitating resistance - when technologies encourage precarity, transience and dynamism, or in a wider context how stitching might gain cultural currency. It is notable that two of the three cases described here are artistic interventions designed to question and critique, and thus not necessarily indicative of the temporalities in mass-consumption digital cartography. Stitching may well be a problematic strategy for the mainstream in an age when tempo has shifted to discourage fixing, in favour of flow. Recent research focusing upon narrative cartography (Caquard, 2013) suggests some of the ways in which memories are increasingly being woven into personal cartographies. 
We suggest that this stand might usefully be developed and extended to new fields beyond the artistic.

A second question that emerges from the strategies described here concerns the affordances that they facilitate (Gibson, 1977; Greeno, 1994). To work, mapping needs to be deployed, read and enacted. But very little research to date has focused on interactionist ecological investigation of how a fixing together of temporal frames might actually be read. Stitched and recombined bricolages, like the artworks produced by Wolfgang Weileder and described by Rachel Wells in chapter 5 of this volume, are challenging to interpret, but paradoxically have to be read if they are to gain cultural currency. We need to find out how memory maps do their work.

A third question arising from the stitching together concerns the implication for spatiality. We would argue that it tends to focus attention on the particular and the unique, and onto places as against spaces. Places, in these stitchings, become holders for memory, moments or rhythms, that might be recombined in a reflective story of mapping with particular and unique resonances. To look at these kinds of practices through a temporal lens does not so much obliterate spatiality, but instead alters the analysis beyond modern conceptions, shifting to a more hybrid understanding of tempo-spatial translations. There is nothing inevitable about flow, and rich place-based analyses are needed to explore how the politics of placeholding emerges in a transient age.

So the capacities of digital mapping to stitch, as against to split, also demand attention. Adam's (2008) notions of tempo, sequence, time frame and rhythm are embodied in critical questions of how stitching can take place in an age when technological context tends to privilege mutability.

\section{(In)formalising}

This section exemplified the various ways in which digital maps shape, predict and invent time. In the introduction to this book, we suggested that technological cartographic projects have either sought to resist inherent temporal instability (such as weather or disease maps), or else sought to build and expand upon it (e.g. various social media-based reaction maps). A tension thus exists between the formalisation of time, and its informal destabilisation. Michiel de Lange's chapter complicates this unstable notion of temporality. Discussing the problematics of the smart city dashboard's 'real-time', he builds on sociologist Barbara Adam's (2008) work to further disassemble the idea of temporal instability. He argues for an asynchronous approach to the city that 'highlights latency, recurrence, deferred understanding and imperfections of the mediating 
process' in mapping the smart city (de Lange, this volume). The other chapters in this section also extend and call into question temporal logics, in and beyond the smart city. Sutherland's work challenges - through the notion of flows the tempo of digital maps. Cate Turk's work on foam ontologies challenges established notions of sequences. Tuur Driesser reverses the temporal modalities present in a PathoMap. Each of them goes beyond Adam's classification to explore processes and relations, and each focuses on different aspects of this (in)formalisation.

As Sutherland has noted in his chapter, the mapping of flows is a wellestablished domain and has come to be associated with more recent global, digital manifestations of capital circulation. The obsession with rendering the world as flow-full (mirroring similar obsessions for the real-time in de Lange's piece, and prediction/prevention in Driesser's), therefore, has led to a curious abandoning of anything fixed, grounded or otherwise stable - despite the continuing presence of infrastructures that facilitate the various flows of capital around the world. Recently, there has been a growing academic interest in the 'logistics' of these flows. That is to say, the systems, architectures and processes that allow for the near-constant movement of goods and people around the world. Ned Rossiter's (2015) work on the global logistics industry and its intertwining with new digital infrastructures and software architectures is particularly relevant in this context.

So, a first key question lies with the materiality of time. If triumphalist narratives proclaiming the free-floating nature of global life are to be avoided, then these nascent interventions are critical. Moreover, things do not move on their own, but are reliant on huge material investments in labour power as well as fixed capital. All too frequently, these aspects are ignored or taken for granted. There is, of course, a temporal specificity to such operations and a requirement that particular activities are carried out in advance of, or just in time for, their use. Think, for example, of global logistics firms such as FedEx, whose current advertising strapline is 'The World on Time'. ${ }^{1}$ Needless to say, there is much more to be done on this logistical time that not only takes account of how this logistical logic plays out, but indeed where it plays out. How these logistical operations are governed, streamlined, standardised and audited by various integrated modules and systems being put to work the world over, literally makes a difference. As is thus evident, there are important material and social concerns beyond Adam's framework, which demand attention.

A second series of questions concerns the conceptual frameworks that might usefully underpin research. These formalised structures themselves strongly influence interpretations of digital mapping. There have been a multitude of academic appraisals of flow-thinking and the mapping of flows, from nodes 
and across networks (see for example Latour, 1986; Deleuze and Guattari, 1988; Castells, 1991). While the taking-up of new conceptual frameworks should always be done with caution, so as to avoid blindly overlaying an otherwise ill-fitting set of ideas on top of already existing practices and processes, Sloterdijk's (1998; 1999; 2004) work on foams presents some interesting opportunities. Indeed, Turk's question of whether 'freezing' phenomena is necessary in order to analyse them - say, in relation to the dynamism of digital mapping interfaces - is richly answered by deploying Sloterdijk. Understanding the way in which various elements combine within a more general architecture, say, in relation to humanitarian projects, is critical. In deploying bubbles and foams, Turk makes a much-needed abstract turn. She implicitly attacks naïve behaviouralist or technicist approaches, frequently seen in new empiricist claims of big data research that privilege technologies (see Kitchin, 2014). But the emphasis on bubbles and foams also critiques Deleuzian or Latourian thought that presupposes a world of networked flow, or a world of nodes and translations. While other theoretical framings stress the 'unfolding' or 'enveloping' properties of time that speak only to a kind of 2D world, Sloterdijk's bubbles and foams point towards a 3D world in which the 'globular' or the 'atmospheric' take centre-stage. The question for future researchers is how conceptual vocabularies might be developed, through which the temporal and the cartographic can be spoken of as being in concert?

A third key question emerges in Tuur Driesser's contribution. How might the future be foreclosed or anticipated in new mapping projects that seek to anticipate emergent risks and situations? While Driesser adopts a novel 'objectoriented' approach - relative to, but not entirely the same as, ANT - there are other nascent avenues that must be considered in light of the ominous suggestion that the contingency of future actions is being shut down (see for example Anderson, 2010). Unlike the material and conceptual provocations above, Driesser probes an intensely political force to speculate on what might be lost - socially, culturally, temporally and cartographically - in light of new biopolitical developments that arguably threaten rather than enhance current ways of living. In this chapter, temporality becomes a new battleground over which political forces exert their will on bodies, objects and relations. Although more optimistic contributions stress that these speculative thoughts are but hopeful 'visions' rather than material realities, the fact remains that they drive a host of actually existing projects and initiatives, many of which are subsumed at present under the 'big data' umbrella embodying a 'capture all' mentality and desiring an 'anticipate it all' future. Perhaps more than anything else, Driesser's intervention seeks to establish the coordinates for this new future-oriented world and as a clarion call for a political consideration of temporalities. 
The authors of (in)formalising have focused not only on the inherent differences between the various temporalities enacted in digital maps, but - and perhaps more crucially - on the troubles of comparing and understanding the nature of such temporalities in relation to one another. The structures beyond time powerfully affect material, conceptual and anticipatory logics enrolled into temporality. Adam's typology significantly underplays these organisational, infrastructural and formal tropes.

\section{It's about time}

This book has taken the reader on a journey through time, revealing ways in which digital mapping can be approached using temporal lenses, from different theoretical positions or methodological ways of doing. May and Thrift (2001) chose to focus on time-space as a series of concepts impacting on wider issues relating to practice across geography, whereas here we emphasise the very particular case of technological change, and the different ways in which a digital remaking of mapping might relate to temporality. The two decades since their intervention have seen significant social, economic and political shifts towards increasingly digital life, with consequent and profound implications for time and temporality. Authors from different interdisciplinary backgrounds have shown what happens when temporality is privileged over spatiality in the analysis of digital mapping. That does not mean that the book sheds the notion of spatiality altogether, but rather that the contributions have mainly worked from tempo-spatiality instead of spatio-temporality.

We have suggested that key research agendas might usefully be progressed by further exploration of ephemeralities and mobilities, stitching and (in)formalising aspects of temporality. Sometimes these suggestions are practical; attending to the mechanisms and affordances of how stitching might occur for example. But changing times also demand more conceptual innovation, such as consideration of how epistemology and formal structures of knowledge impact researching temporalities of digital mapping. The theories and methodologies that emerged through these very different interventions can help us to refine and extend our understanding of temporality in relation to digital mapping and digital culture in general. It is about time, because, as the contributions of this book show, digital mapping interfaces are making new temporal experiences that hitherto have not been sufficiently addressed by approaches to mapping that emphasise spatiality of the medium. It is about time, because mapping has begun to be re-thought in productive temporal terms. 


\section{Note}

1 See: http://i1 108.photobucket.com/albums/h420/Dr3am3r1011/IMG_2719.jpg (accessed 4 December 2017).

\section{References}

Adam, B. (2008) Of timespaces, futurescapes and timeprints. [Online] Available at: www. cardiff.ac.uk/socsi/futures/conf_ba_lueneberg170608.pdf (accessed 1 August 2016).

Anderson, B. (2010) Preemption, precaution, preparedness: Anticipatory action and future geographies. Progress in Human Geography, 34(6): pp. 777-798.

Caquard, S. (2013) Cartography: Mapping narrative cartography. Progress in Human Geography, 37(1): pp. 135-144.

Castells, M. (1991) The Informational City: Information Technology, Economic Restructuring, and the Urban-Regional Process. Oxford: Blackwell.

Deleuze, G. and Guattari, F. (1988) A Thousand Plateaus: Capitalism and Schizophrenia. London: Bloomsbury Publishing.

Elsaesser, T. (2009) Between Erlebnis and Erfahrung: Cinema experience with Benjamin. Paragraph, 32(3): pp. 292-312.

Gibson, J. J. (1977). 'The theory of affordances'. In: Shaw, R. and Bransford, J. (eds) Perceiving, Acting, and Knowing: Toward an Ecological Psychology. Hillsdale, New Jersey: Erlbaum, pp. 67-82.

Greeno, J. G. (1994) Gibson's affordances. Psychological Review, 101(2): pp. 336-342.

Gregg, M. (2015) Inside the data spectacle. Television and New Media, 16(1): pp. 37-51.

Kitchin, R. (2014) Big data, new epistemologies and paradigm shifts. Big Data and Society. [Online] Available at: http:/ /bds.sagepub.com/content/spbds/1/1/2053951 714528481.full.pdf (accessed 31 August 2016).

Kitchin, R. and Dodge, M. (2007) Rethinking maps. Progress in Human Geography, 31(3): pp. 331-344.

Latour, B. (1986) 'Visualization and cognition: Thinking with eyes and hands'. In: Kuklick, H. (ed.) Knowledge and Society: Studies in the Sociology of Culture Past and Present Volume 6. New York: Jai Press, pp. 1-40.

Massey, D. (1991) A global sense of place. Marxism Today, June: pp. 24-29.

May, J. and Thrift, N. (eds) (2001) Timespace: Geographies of Modernity. London: Routledge.

Olsson, G. (2007) Abysmal: A Critique of Cartographic Reason. Chicago, Illinois: University of Chicago Press.

Rossiter, N. (2015) Coded vanilla: Logistical media and the determination of action. South Atlantic Quarterly, 114(1): pp. 135-152.

Sloterdijk, P. (1998) Sphären I-Blasen. Frankfurt: Suhrkamp.

Sloterdijk, P. (1999) Sphären II - Globen. Frankfurt: Suhrkamp.

Sloterdijk, P. (2004) Sphären III - Schäume. Frankfurt: Suhrkamp. 
Thatcher, J. (2014) Living on fumes: Digital footprints, data fumes, and the limitations of spatial big data. International Journal of Communication, 8: pp. 1765-1783.

Verhoeff, N. (2012) Mobile Screens: The Visual Regime of Navigation. Amsterdam: Amsterdam University Press.

Wilmott, C. (2016) Small moments, big data: Mobile mapping in everyday life. Big Data and Society, 3(2). https://doi.org/10.1177/2053951716661364.

Wood, D. (1992) The Power of Maps. New York: Guilford Press. 